\title{
The effect of graphene network formation on the electrical, mechanical, and multifunctional properties of graphene/epoxy nanocomposites
}

Arnaud Kernin ${ }^{a, \#}$, Kening Wan ${ }^{a, \#}$, Yi Liu ${ }^{a, b}$, Xuetao Shic, Jie Kongc, Emiliano Bilotti, ${ }^{\mathrm{a}, \mathrm{b}}$, Ton Peijs ${ }^{a, b, c, *}$, Han Zhang ${ }^{a, b, c, *}$

a School of Engineering and Materials Science, and Materials Research Institute, Queen Mary University of London, Mile End Road, E1 4NS London, UK

b Nanoforce Technology Ltd., Joseph Priestley Building, Queen Mary University of London, Mile End Road, E1 4NS London, UK

c NPU-QMUL Joint Research Institute of Advanced Materials and Structures, Northwestern Polytechnical University, Xi'an, 710072, P. R. China

Abstract: The network formation of reduced graphene oxide ( $\mathrm{GO}$ ) within an epoxy resin during the curing process has been in-situ visualised for the first time, with its effect on electrical, mechanical, and multifunctional properties of these nanocomposites explored. Different initial states of dispersion and filler contents were employed to examine the nanofiller network formation process. Good electrical conductivity $\left(10^{-3} \mathrm{~S} / \mathrm{m}\right.$ at $\left.0.05 \mathrm{wt} . \% \mathrm{rGO}\right)$ together with good mechanical reinforcement (12\% increase in flexural modulus at $0.2 \mathrm{wt} . \%$ rGO) were obtained at relatively low filler loadings. The integrated strain sensing capabilities based on the rGO network were explored with good sensitivity and repeatability. Joule heating was performed as a potential application for de-icing of multifunctional composite components with good heating capability from $-20^{\circ} \mathrm{C}$ to $20^{\circ} \mathrm{C}$ within $2 \mathrm{~min}$.

Keywords: graphene, epoxy, percolation, electrical conductivity, mechanical properties, sensing, de-icing.

\footnotetext{
* Corresponding author. Tel.: +44 02078828865 (T.Peijs); +44 02078822726 (H.Zhang)

E-mail address: t.peijs@qmul.ac.uk; han.zhang@qmul.ac.uk

\# A.K. and K.W. contributed equally to this work.
} 


\section{Introduction}

The use of graphene and various forms of graphene nanoplatelets (GNPs) as nanofillers for polymers has been explored extensively over the last few years due to their potential to improve electrical, mechanical, thermal, and other multifunctional properties of matrix materials at relatively low filler loadings. Epoxy, one of the most widely used matrix materials for engineering composites, possesses advantages of high stiffness, good chemical resistance but also limitations of relatively high brittleness and poor electrical and thermal performances. Great progress has been made to improve these drawbacks of epoxy resins without sacrificing their original performance [1-3], by introducing nanofillers such as carbon nanotubes (CNTs) [4], reduced graphene oxide (rGO) [5], graphene nanoplatelets (GNPs) [6-8] or some of their hybrids [9]. Rafiee et al. reported an increased Young's modulus and tensile strength of $31 \%$ and $40 \%$, respectively, with as little as $0.1 \mathrm{wt} . \%$ GNPs in epoxy [10]. Li et al. obtained a $160 \%$ increase in flexural modulus with 4 wt.\% in-situ exfoliated GNPs in epoxy matrix, and an electrical conductivity of $10^{-2} \mathrm{~S} / \mathrm{m}$ at $3 \mathrm{wt} . \%$ filler [11]. Monti et al. compared two different solvent-assisted methods to disperse GNPs into epoxy and found that dispersing GNPs in epoxy monomer rather than hardener results in better nanofiller dispersion and electrical percolation thresholds between 1-2 \% together with an increase in glass transition temperature $\left(T_{g}\right)$ [12]. With integrated electrical conductivity, multifunctional epoxy based nanocomposites have also been successfully fabricated for strain/damage sensing and electromagnetic interference (EMI) shielding properties and utilised for continuous fibre reinforced composites [13-17].

Although great progress has been made with many promising results, very little attention has been paid to the graphene network formation process during epoxy curing and subsequent effects on final properties of the obtained nanocomposites [18, 19]. Tang et al. investigated 
the effect of quality of graphene dispersion on mechanical properties of epoxy nanocomposites and found a higher $\mathrm{T}_{\mathrm{g}}$ together with higher electrical and mechanical properties for well dispersed nanocomposites in contrast to poorly dispersed systems [18]. Network formation of CNTs has been explored in both thermoset [20, 21] and thermoplastic systems $[22,23]$, including the use of in-situ monitoring of electrical conductivity during the network formation and re-aggregation process in thermoplastics. With the correct design of processing parameters, it was shown that electrical conductivity can be increased by as much as 9 orders of magnitude for the same amount of filler using this re-agglomeration or so-called dynamic percolation process $[22,23]$. In addition, Bilotti et al. demonstrated a simplified relationship that linked polymer viscosity to nanofiller network formation and was able to predict electrical conductivity based on Arrhenius type behaviour of polymer melts, providing a simple tool to predict network formation based on differences in polymer matrix viscosity [24]. Battisti et al. developed an on-line dispersion monitoring system by measuring electrical resistivity of CNTs in liquid polyester pre-polymer, although it was found that the electrical behaviour can be affected by CNT network formation rather than solely by dispersion state [25]. Clearly, a similar re-aggregation process for graphene nanofillers within thermoset matrices can be expected during the curing process of thermosetting resins. In fact, it is expected that this process is operative at much shorter timescales given the much lower viscosity of such resins compared to thermoplastics.

It is generally acknowledged that a homogeneous dispersion of nanofillers is required for good mechanical reinforcement, while a slightly agglomerated filler network is needed to create a percolated electrically conductive network at low filler loadings [26-28]. Obviously, well dispersed graphene nanofillers will tend to re-agglomerate during the curing of epoxy resins as a result of their large surface areas, affecting their final morphology and associated 
performance. However, no previous work has been performed to visualise the actual network formation process of graphene nanofillers or examined the associated effects on electrical, mechanical, and other multifunctional properties. Clearly, insight in such network formation process is extremely important for providing guidelines for the fabrication of nano-engineered composites with desired multifunctional properties.

In this work, the graphene network formation process within a liquid epoxy resin during curing has been successfully visualised and monitored by optical microscopy for the first time, providing direct evidence to correlate different initial and re-agglomerated dispersion levels with observed electrical and mechanical properties. Electrical conductivity was introduced at ultra-low rGO concentrations ( $0.05 \mathrm{wt} . \%)$ as a result of a good level of initial dispersion and a subsequent re-agglomeration process, while simultaneously increasing the flexural modulus by $12 \%$ at 0.2 wt.\% loadings. Multifunctional properties such as electrical strain sensing have been characterised with high levels of sensitivity (gauge factor 41 at $0.2 \mathrm{wt} . \% \mathrm{rGO}$ ). Potential applications based on these multifunctional resin systems have been explored through a simple demonstrator involving a de-icing nanocomposite based on the Joule heating effect.

\section{Experimental}

\subsection{Materials}

Mono-layer reduced graphene oxide ( $r G O)$ with a D50 value (according to supplier) of $40 \mu \mathrm{m}$ was supplied by Avanzare (Spain) (SG-221) and used as received. The two components low viscosity epoxy resin RX771C (bisphenol A diglycidyl ether, DGEBA) and HX932C (aromatic amine) were supplied by Robnor ResinLab (UK) and mixed at a weight ratio of 100:24, with the viscosity values of $600 \mathrm{mPa}$.s.

\subsection{Dispersion and exfoliation of rGO in epoxy resin}


Three different dispersion strategies were applied to prepare rGO/epoxy nanocomposites with various filler concentrations: (i) low shear simple mixing using a high-speed homogenizer (IKA T-25) to mix the desired amount of rGO within epoxy resins at $1000 \mathrm{rpm}$ for $10 \mathrm{~min}$; (ii) medium shear mixing using a three roll mill (TRM) (80E EXAKT GmbH, Germany) at a fixed gap distance of $15 \mu \mathrm{m}$ and $5 \mu \mathrm{m}$ for back and front, respectively, and at a constant apron roll speed of 200 rpm for four cycles; (iii) high shear mixing using TRM with progressively reduced gap distances $(90 / 30,60 / 20,45 / 15,30 / 10$, and $15 / 5 \mu \mathrm{m}$ for back and front gaps, respectively) at constant apron roll speed of $200 \mathrm{rpm}$ for ten cycles, followed by a force mode setting of 3 $\mathrm{N} / \mathrm{mm}$ for two cycles to provide further exfoliation of the graphene filler, as previously reported in $[8,29]$.

\subsection{Fabrication of rGO/epoxy nanocomposites}

After the dispersion/exfoliation process, hardener was added to the rGO/epoxy mixture at a ratio of 100:24 w/w. Degassing was performed at $80^{\circ} \mathrm{C}$ for $1 \mathrm{~h}$ under vacuum and continuous stirring before the mixture was poured into a vertically positioned steel mould with a cavity of $120 \times 75 \times 3 \mathrm{~mm}^{3}$ for curing. The performed curing cycle was isothermal at $120^{\circ} \mathrm{C}$ for $12 \mathrm{~h}$, as suggested by the supplier. The sample was cured and cut horizontally along the $75 \mathrm{~mm}$ width using a diamond cutting wheel to avoid any sedimentation effects on measured properties, as the average value of specimens from different locations across the height was used.

\subsection{Characterisations}

\section{Optical microscopy}

The visualisation of rGO network formation during epoxy curing was performed under an optical microscope (VWR TR300) in combination with a Linkam HFS600 heating stage. For each specimen, $10 \mu \mathrm{l}$ of rGO/epoxy sample was taken from the degassed mixture and placed onto a pre-heated $\left(120^{\circ} \mathrm{C}\right)$ glass slide using an Eppendorf pipette. A thin glass lid was placed on top 
of the resin droplet before observation. The video was taken from the moment that the glass lid was placed, and marked as time zero. Images at different time intervals were taken from the recorded videos.

Scanning electron microscopy

Fracture surfaces were obtained after cyro-fracturing the cured specimen, and examined by scanning electron microscopy (SEM) (FEI Inspect-F, Netherlands). Images at different magnifications were taken in order to examine the final rGO network morphology after curing. $X$-ray diffraction

X-ray diffraction (XRD) (Philips PW 3830, Netherland) was used to characterize the reduction level of $\mathrm{rGO}$ powder. X-ray texture scans were obtained between $2 \theta=5-70^{\circ}$ at a scanning rate of $1 \%$ min.

\section{Electrical properties}

The electrical conductivity of various specimens were measured by two-point measurements using a voltage power source (Agilent, 6614C) and a picoammeter (Keithley, 6485). Specimens with dimensions of $3 \times 12.7 \times 70 \mathrm{~mm}^{3}$ were measured lengthwise in order to obtain more reliable conductivity values to avoid inaccurate readings from localised networks rather than macroscopically dispersed nanofillers. Silver paint was applied to both ends of the specimens to reduce contact resistance.

\section{Mechanical properties}

Flexural tests were performed in accordance with ASTM D790 standard and specimen dimensions of $3 \times 12.7 \times 70 \mathrm{~mm}^{3}$. The cross-sectional surface was polished after cutting. The span-to-depth ratio was kept 16 to 1 for all three-point bending specimens, with the calculated crosshead speed being equivalent to a strain rate of $0.01 \mathrm{~min}^{-1}$ at the outer surface in accordance with standard. 


\section{Strain sensing}

Electrical strain sensing performance was characterised by monitoring the electrical resistance variation upon applied strain under flexural loading. The electrodes were attached at the tensile surface of the specimen after carefully polishing the areas to remove the outer epoxy layer. Two test configurations were used; one close to the centre of the beam with a distance of $20 \mathrm{~mm}$ between the two electrodes (mid-electrodes), or one directly from the ends of the specimens with a distance of $70 \mathrm{~mm}$ (end-electrodes) between the electrodes. Constant voltage of $10 \mathrm{~V}$ was applied throughout the test. The gauge factor (GF) was calculated based on the obtained resistance change over the outer surface strain.

Thermal (Joule) heating

Two test environments with a temperature of $-20{ }^{\circ} \mathrm{C}$ and room temperature (RT) were employed to examine potential de-icing and heating capabilities of the nanocomposite samples. A power supply (GE EPS 301) was set to $240 \mathrm{~V}$ to simulate the standard voltage for both cases. Thermocouples were attached to the surface of a cast panel with a voltage of 240 V applied after $30 \mathrm{~s}$ for all tests. A thermal infrared camera (FLIR E40, UK) was used to examine the homogeneity of heating after $120 \mathrm{~s}$ of heating from RT.

\section{Results and discussions}

\subsection{As-received graphene characterisation}

The as-received reduced graphene oxide ( $\mathrm{rGO}$ ) powders were examined under SEM and XRD as shown in Fig. 1. A very porous structure can be seen in the SEM images with a lateral size of around $30 \mu \mathrm{m}$. The XRD pattern of the as-received rGO powder shows a relatively broad characteristic $\left(\begin{array}{lll}0 & 0 & 2\end{array}\right)$ peak at $26.5^{\circ}$, which corresponds to a " $d$ " spacing value of $0.336 \mathrm{~nm}$, indicating a good reduction from graphene oxide $(\mathrm{GO})$ to reduced graphene oxide ( $\mathrm{rGO}$ ). 

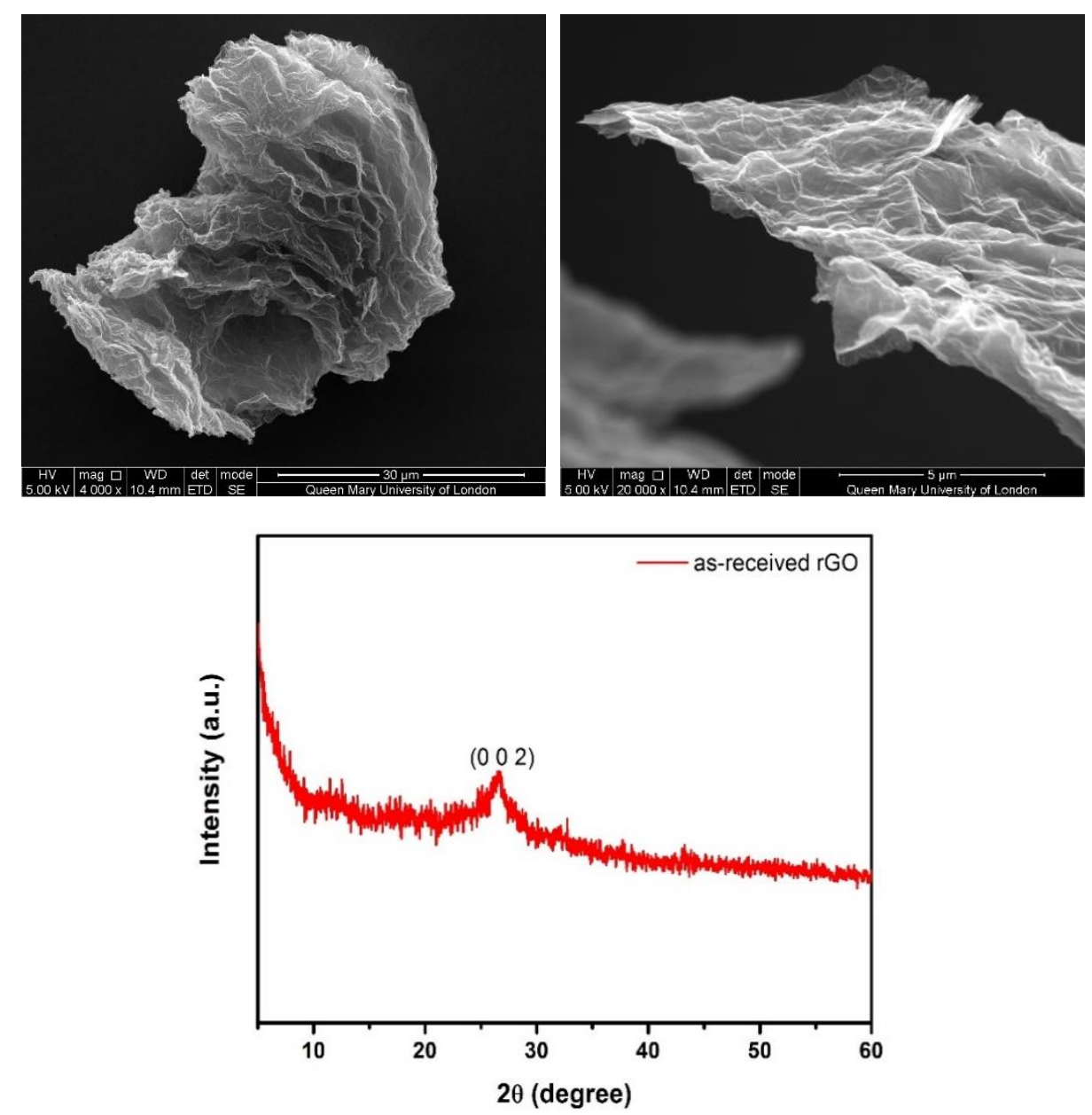

Fig. 1 SEM images of as-received rGO fillers, showing lateral dimensions of around $30 \mu \mathrm{m}$ and a porous structure (top); the XRD scan indicates a good level of reduction (bottom).

\subsection{Visualisation of graphene network formation}

The initial level of graphene dispersion plays an important role in the final network formation and hence on resulting properties. Fig. 2 shows the rGO network formation process within the liquid epoxy resin during curing after two distinct processing conditions: medium shearing (with fixed gap and less cycles during TRM) and high shearing (with progressively increased high shearing force during TRM). Three representative concentrations of 0.05 wt.\%, 0.2 wt.\%, and 0.5 wt.\% were selected with the aim of exploring the establishment of a graphene nanofiller network at various loading levels.

For an ultra-low graphene concentration of $0.05 \mathrm{wt} . \%$ after high shearing, a homogeneous distributed graphene morphology without any filler network connections was found at the 
beginning of curing (time $0 \mathrm{~s}$ ), followed by very slow filler migration with no obvious microscopic change until $50 \mathrm{~s}$. At a time interval of $3 \mathrm{~min}$, few areas of isolated networks within the observed region can be observed, with a continued slow migration of smaller particles in surrounding areas. After $10 \mathrm{~min}$, a clear filler path is observed based on re-agglomerated graphene, which remains macroscopically similar in shape until full cure after $12 \mathrm{~h}$.

With increased graphene content (0.2 wt.\%) after high shear TRM mixing, an obvious increase in volume fraction of graphene can be found at time zero, followed by very quick network formation after $3 \mathrm{~s}$. Initial network formation can already be observed in optical microscopy images after $10 \mathrm{~s}$, with a further enhancement towards a more robust network after $50 \mathrm{~s}$. No obvious changes can be seen from 3 min onwards until the end of curing due to the already strong network formed at the very beginning of cure. When the filler concentration is further increased ( $0.5 \mathrm{wt} . \%)$, the entire area of observation has been occupied by graphene fillers, with no obvious indication of further network formation during curing since a percolated network was already established at the very beginning of cure. Clearly an optimum amount should be aimed for in order to reach a desired network and associated properties at minimum cost. Regarding the medium shear TRM mixed specimens, a less homogeneous distribution was found with evidence of few small agglomerates at time zero in comparison to high shear TRM mixing at similar concentrations. This is attributed to the lower shear force employed during the dispersion process. As a result, local network formation occurred at a higher rate for medium-shear mixed specimens, especially in regions where initial agglomerates exist. However, to obtain a percolated network at macroscopic level, a more homogeneous initial filler distribution is required as shown after high shear processing. 


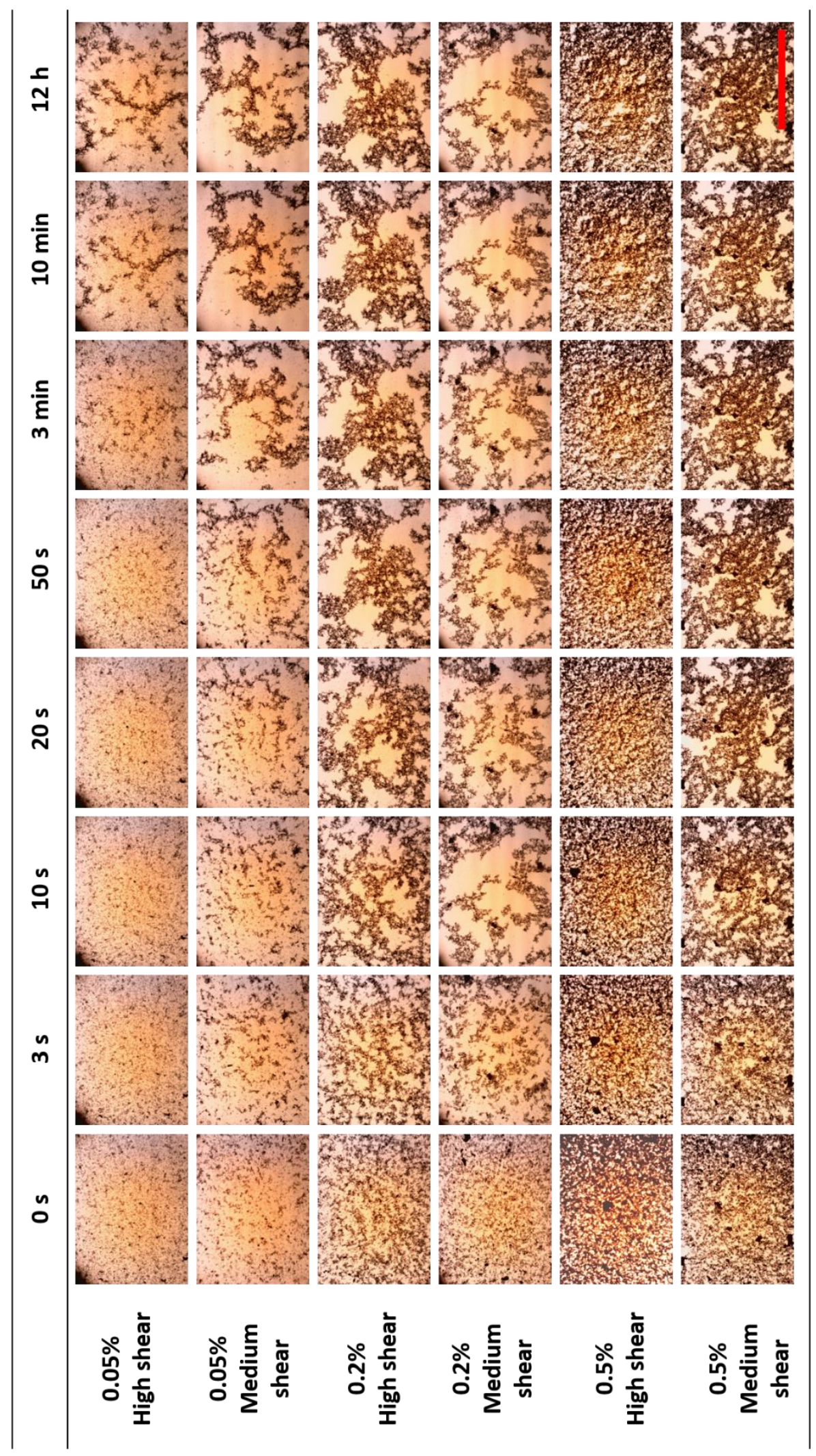

Fig. 2 The graphene network formation process after two different (medium and high shear) TRM processing conditions, showing clear re-agglomeration and network formation processes of rGO during epoxy curing. Clear conductive pathways were found at extremely low rGO concentrations after 10 min of curing. (Scale bar at bottom right represents $1 \mathrm{~mm}$ ). 
It has been previously reported that conductive filler network formation within a thermoplastic polymer matrix is mainly governed by the melt viscosity of the polymer matrix, which is a first order thermally activated process [24]. With a reduced viscosity of the polymer melt, a reduction in activation energy can be expected, leading to a faster Arrhenius-type network formation process. Hence, compared to for example earlier reported thermoplastic polyurethane (TPU) systems with melt viscosities in the range of $10^{3}$ to $10^{4} \mathrm{~Pa} . \mathrm{s}$ and a required annealing (or network formation) time to reach high levels of electrical conductivity of more than a few hundred seconds [24], much shorter times can be expected for the current epoxy resin system with a measured viscosity of only $3.1 \times 10^{-2} \mathrm{~Pa} . \mathrm{s}$ at $80^{\circ} \mathrm{C}$. This orders of magnitude reduced viscosity leads to greatly reduced activation energies $\left(53 \mathrm{~kJ} \mathrm{~mol}^{-1}\right.$ for epoxy from 249 $\mathrm{kJ} \mathrm{mol}^{-1}$ for TPU [24], see SI) and as a result much shorter network formation times. This is confirmed by the current epoxy system where only $10 \mathrm{~s}$ was required to establish a percolating network from an initially dispersed state for 0.2 wt.\% rGO, i.e. an orders of magnitude reduction in network formation time as compared to the reported thermoplastic system [24]. It is easy to understand that graphene network formation and epoxy crosslinking are opposing dynamic processes. However, it can be expected that for normal curing of liquid epoxy resins the curing time will not greatly affect the filler network formation. As long as initial dispersions are homogeneous and no ultra-fast curing epoxies are used a clear network formation process can be expected even at the very early stage of cure.

\subsection{Scanning electron microscopy}

The morphologies of the various rGO/epoxy specimens were examined by SEM after cyrofracturing, in order to evaluate the final graphene network after curing. In the case of lowshear dispersion methods (high-speed homogenizer), large agglomerates with stacked graphitic platelets were found for all concentrations, including $0.05 \mathrm{wt} . \% \mathrm{rGO}$. This is due to 
the insufficient level of initial dispersion from this simple mixing process. For medium shear TRM dispersion methods a much finer filler dispersion was found at low filler contents $(0.05$ wt.\%), with clear evidence of percolated networks at a concentration of $0.2 \mathrm{wt} . \% \mathrm{rGO}$. No obvious morphological changes from $0.2 \mathrm{wt} . \%$ to $0.5 \mathrm{wt}$.\% occurred apart from more graphene appearing within the observed region. For high shear TRM processed specimens with good initial levels of dispersion and smaller initial particle dimensions (as shown in Fig. 2), traces of connected filler networks can already be observed at an ultra-low rGO loading of 0.05 wt.\%. For $0.2 \mathrm{wt} . \% \mathrm{rGO}$ in epoxy, a more robust and connected network is found in comparison to well dispersed nanocomposites. However, with further increasing rGO content ( 0.5 wt.\%) and high shear processing a more fragmented morphology is observed, with much smaller segmentations covering the complete area. This morphology can be attributed to the high shear force induced by the TRM exfoliation process at higher filler loadings, as these loadings increase the resin viscosity dramatically, resulting in even higher shearing forces and breakup of the rGO into smaller particles laterally.

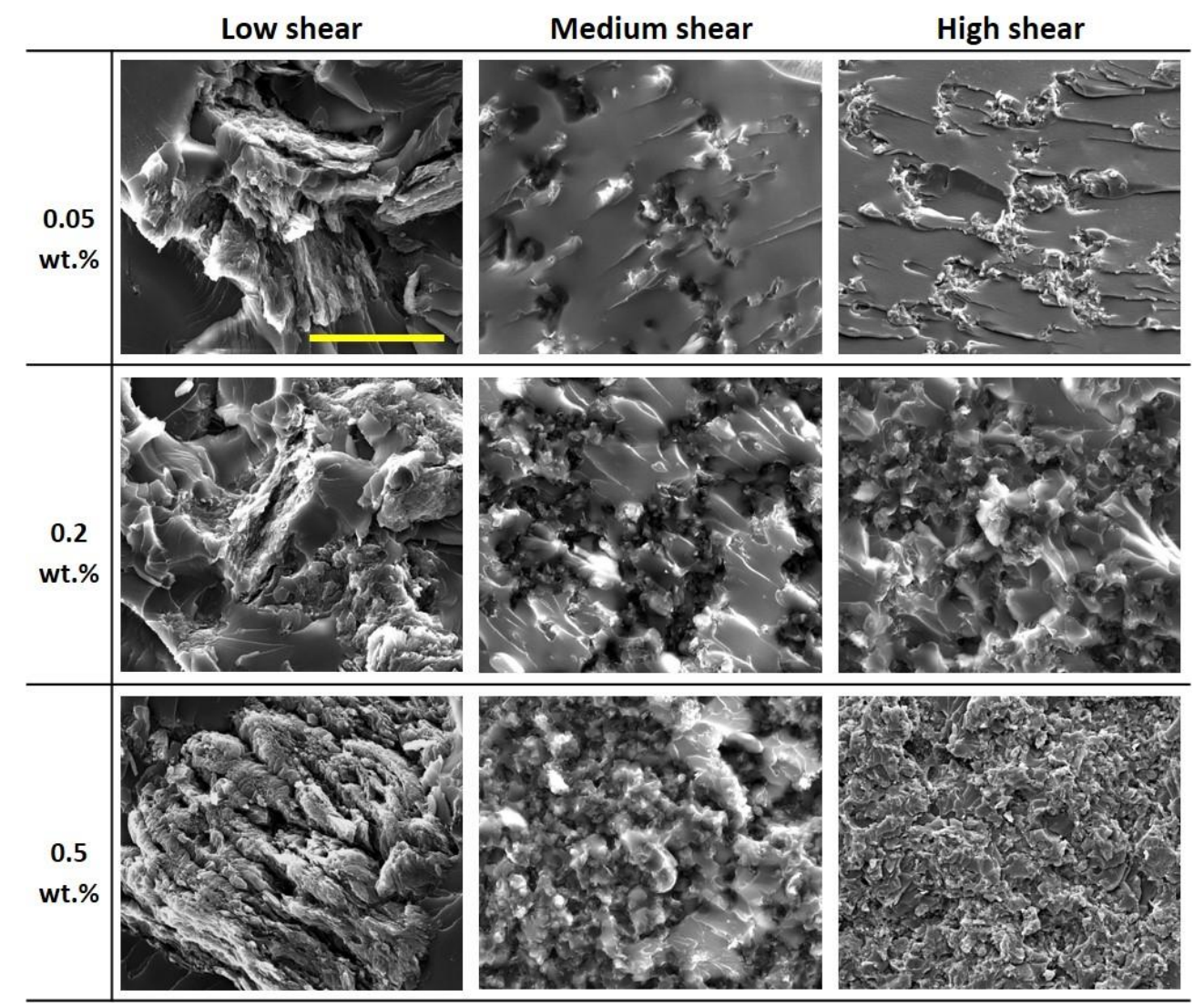


Fig. 3 SEM images of rGO/epoxy nanocomposites prepared with three different filler concentrations and three processing conditions: low shear (simple mixing); medium shear (TRM dispersed); and high shear (TRM exfoliated). Scale bar represents $50 \mu \mathrm{m}$. At the same filler concentration a clear difference in graphene networks can be observed between these three methods, with the high shear process inducing a homogeneous initial dispersion essential for nanofillers to build up a percolating network upon epoxy curing at low filler loadings.

\subsection{Electrical properties}

In order to obtain a high level of electrical conductivity at relatively low filler loadings, two important steps with regards to network formation aspects are required: (i) an initial homogeneous dispersion of nanofillers within the resin; (ii) a dynamic percolation process that involves the formation of a conductive network through re-agglomeration. As described by classic percolation theory, no electrical conductivity can be measured before the filler loading reaches the percolation threshold, which is the lowest amount of conductive filler required to build up a connecting pathway for electrons to travel through the specimen. Clearly, higher aspect ratio (length over thickness) nanofillers are favoured to establish such pathways at a low percolation threshold $[30,31]$. Once a percolated network is formed within the matrix, the electrical conductivity value will still increase with increasing amount of fillers but at a much reduced rate and a final plateau is reached in terms of conductivity values.

Fig. 4 shows the electrical conductivity values of $\mathrm{rGO} / \mathrm{epoxy}$ nanocomposites with different concentrations under low, medium and high shear processing. Surprisingly, a measurable electrical conductivity at the level of $10^{-3} \mathrm{~S} / \mathrm{m}$ was obtained after high shear mixing and a filler loading as low as $0.05 \mathrm{wt} . \%$, which is comparable to previously reported ultra-low percolation values for graphene/epoxy systems $[32,33]$. This is attributed to the high shear force induced by the TRM process, leading to a good initial dispersion (as shown in Fig. 2) followed by a dynamic percolation process to form network connections, as confirmed by the SEM images 
in Fig. 3. Above the percolation threshold the electrical conductivity increases slowly with increasing amount of filler, finally reaching a value of $9.1 \times 10^{-2} \mathrm{~S} / \mathrm{m}$ at $0.5 \mathrm{wt} . \% \mathrm{rGO}$ loading. After medium shear TRM processing, no electrical conductivity can be measured for 0.05 wt.\% rGO and the current sample length of $70 \mathrm{~mm}$, while a measureable value slightly below $10^{-3}$ $\mathrm{S} / \mathrm{m}$ was obtained for $0.1 \mathrm{wt} . \% \mathrm{rGO}$. Above the percolation threshold very similar electrical conductivity values were obtained for both medium- and high shear processed nanocomposites, which is consistent with previous morphological findings of percolated rGO networks (see Fig. 2).

For low shear processing (high-speed homogenizer), no electrical conductivity can be measured until 0.2 wt.\% with only a conductivity level of $10^{-4} \mathrm{~S} / \mathrm{m}$ which is much lower than medium and high shear TRM processed specimens. Even at the highest loading of $0.5 \mathrm{wt} . \%$ in the current study, the electrical conductivity just reached the $10^{-3} \mathrm{~S} / \mathrm{m}$ level which is almost two orders of magnitude lower than for the other two TRM based mixing processes. This difference is attributed to the lack of shear induced kinetic energy, being insufficient for dispersion and resulting in large aggregates and stacked layers between rGO fillers, as confirmed by the SEM images in Fig. 3.

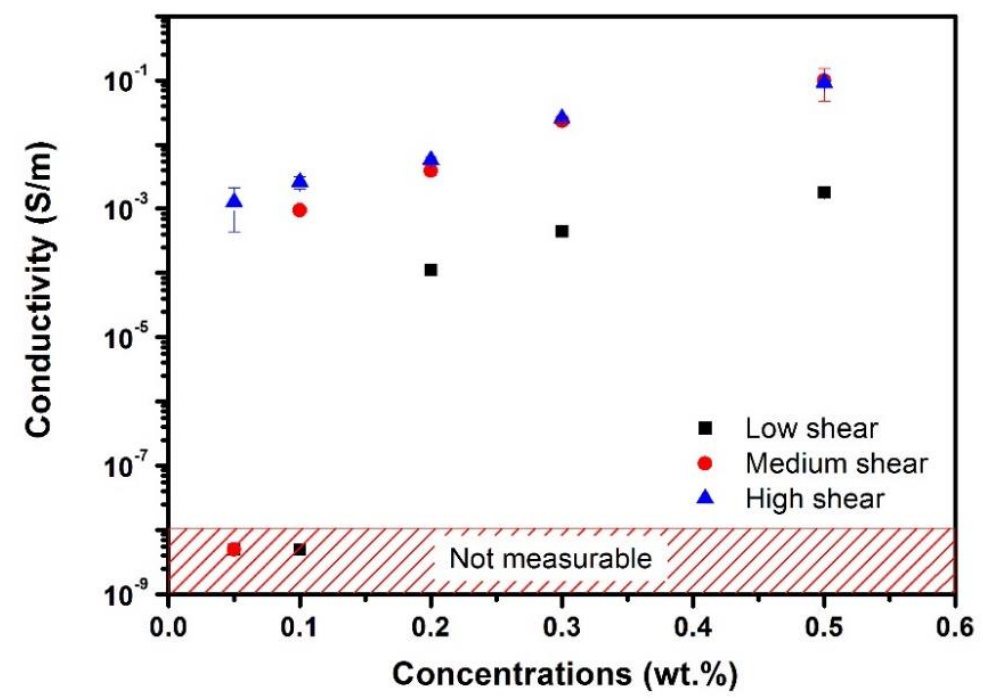

Fig. 4 Electrical percolation curves for rGO/epoxy nanocomposites prepared by three different processing conditions, showing an extremely low percolation threshold especially for high-and medium shear TRM processed specimens. 


\subsection{Mechanical properties}

Fig. 5 shows the flexural properties of rGO reinforced epoxy at different concentrations. The dotted line indicates a flexural modulus of $2.92 \mathrm{GPa}$ as measured for neat epoxy without nanofiller. Clearly with the addition of rGO up to $0.5 \mathrm{wt} . \%$, most specimens show an increase in flexural modulus in comparison to the reference specimen, except of low shearing dispersed specimens with low rGO loadings due to their insufficient dispersion. At low filler loadings, an increasing trend in mechanical reinforcement is observed for increasing levels of shearing as a result of improved levels of exfoliation and more homogeneous graphene dispersions.

The highest flexural modulus (3.28 GPa) was obtained for 0.2 wt.\% rGO/epoxy nanocomposites after high shear TRM processing, with a more than $12 \%$ modulus increment in comparison to the reference specimen. Rafiee et al. reported a 31\% increase in Young's modulus with 0.1 wt.\% rGO which was actually higher than their theoretical prediction [10]. This discrepancy might be due to possible changes in crosslink density of the epoxy resin after the addition of rGO and/or orientation of the platelets. To exclude the first effect, glass transition temperatures $\left(\mathrm{T}_{\mathrm{g}}\right)$ were measured for all nanocomposites, revealing no obvious changes in $\mathrm{T}_{\mathrm{g}}$ with different $\mathrm{rGO}$ loadings (see SI Table S-1), suggesting that changes in crosslink density did not significantly contribute to the observed increase in elastic modulus. The increment obtained in this study is comparable to reported enhancements in mechanical properties with low graphene contents and believed to be due to the initial homogeneous dispersion and formation of a three-dimensional percolated network after the high shear exfoliation process. Exfoliation also increases the total surface area of the nanoplatelets, leading to a more efficient mechanical reinforcement. This current mechanical reinforcement of $12 \%$ at 0.2 wt.\% rGO was further assessed using the Halpin-Tsai micromechanical model with a Mori-Tanaka modified shape factor, assuming a random 3D filler orientation (see SI) and yielding a back-calculated effective filler modulus of $680 \mathrm{GPa}$ for rGO. This value sits well between the elastic modulus of $\mathrm{GO}(\sim 250 \mathrm{GPa})[34,35]$ and pristine graphene ( 1 TPa) [36], 
confirming the high reinforcing efficiency of this $\mathrm{rGO} /$ epoxy system. With increasing rGO filler content and high shear TRM processing, the elastic modulus first increased from $3.05 \mathrm{GPa}$ at 0.05 wt.\% to $3.28 \mathrm{GPa}$ at $0.2 \mathrm{wt} . \%$, followed by a descending trend for $\mathrm{rGO}$ loadings from 0.3 wt.\% to 0.5 wt.\%. Apart from aggregates at higher filler loadings acting as internal defects, this trend is believed to be due to excessive shearing of these viscous $\mathrm{rGO}$ /epoxy systems, resulting in break-up of rGO platelets and reduced lateral dimensions. This was confirmed by the fractography study of 0.5 wt.\% rGO/epoxy nanocomposites where a fragmented morphology was observed in these composites (see Fig. 3).

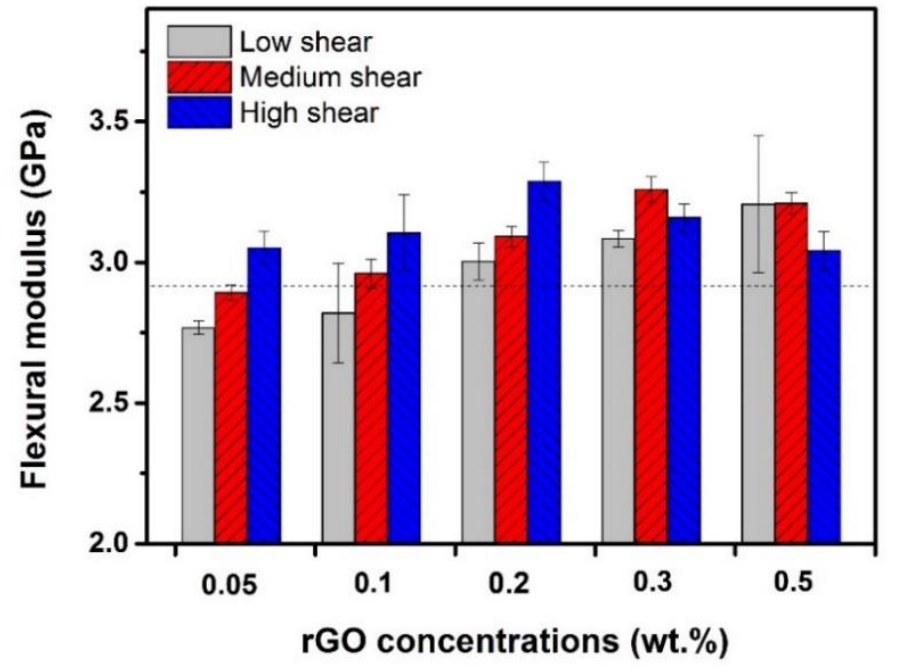

Fig. 5 Flexural modulus of rGO/epoxy nanocomposites prepared by three different processing methods, showing good reinforcement at extremely low filler concentrations (0.05 wt.\%) in the case of high shear TRM processing. The dashed line at 2.92 GPa indicates the baseline modulus of neat epoxy without any filler.

For medium shear TRM mixed specimens, an increasing trend in flexural modulus was found with increasing rGO content, reaching a maximum of $3.26 \mathrm{GPa}$ at $0.3 \mathrm{wt} . \%$. Compared to high shear mixed specimens a similar or slightly reduced value (3.21 GPa) was found above this optimal concentration. For the low shear (high-speed homogenizer) mixed specimens, much lower levels of reinforcement were found for all concentrations apart from the $0.5 \mathrm{wt} . \%$ specimens while a large variation in properties was associated with the presence of large 
aggregates, acting as defects within the matrix. It is well established that when the filler loading is too high in nanocomposites, the possibility of agglomeration as well as restacking between rGO increases dramatically, resulting in a less efficient mechanical reinforcement. This minor reduction in mechanical performance with further increased filler loadings is also consistent with previous studies [27, 37-40].

\subsection{Strain sensing properties}

With the induced electrical conductivity, the rGO/epoxy nanocomposites can be used as a multifunctional materials to detect various external stimuli such as mechanical deformation. Although electrical conductivity was already successfully introduced with as little as 0.05 wt.\% rGO in epoxy, 0.1 wt.\% rGO/epoxy composites were initially chosen for this strain sensing study in order to attain a more stable and clear strain sensing signal based on a more robust rGO network. Two test configurations were evaluated with electrodes either positioned at the bottom (tension) surface or ends of the specimen as illustrated in Fig. 6 a.

Fig. $6 c$ and d show the electrical resistance change in correlation with the applied outer surface strain in the flexural specimens for the two test configurations. For $0.1 \mathrm{wt} . \%$ rGO in epoxy (Fig. $6 c)$, electrical resistance was first reduced with an applied strain up to $0.5 \%$, then returned back to its original level upon unloading. A clear trend was observed under cyclic loading, with a certain level of variation in measured resistance. Although the electrodes were attached to the tension surface of the flexural specimens, a reduction in resistance upon loading was obtained which can be attributed to a reduction in inter-particle distance between rGO nanofillers at the compressive side of the sample (as illustrated in Fig. 6b). This finding is consistent with previous sensing literature under flexural loadings [41, 42]. The calculated gauge factor (GF) for the end-electrodes configuration (33.5) is much higher than for the mid surface electrodes (19) due to accumulative tunnelling effects between internal rGO networks. 
As expected, a much clearer sensing signal was found for $0.2 \mathrm{wt} . \% \mathrm{rGO} / \mathrm{epoxy}$ specimens under the same cyclic loading due to the presence of a more robust and stable internal conductive network. A high and stable gauge factor (GF) of 41.5 was obtained which can be used for strain monitoring and structural health monitoring of epoxy based composites with a good level of sensitivity and repeatability.

(a)

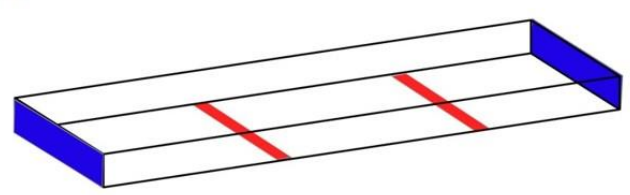

Electrode positions on bottom surface (under tension) and two ends

(c)

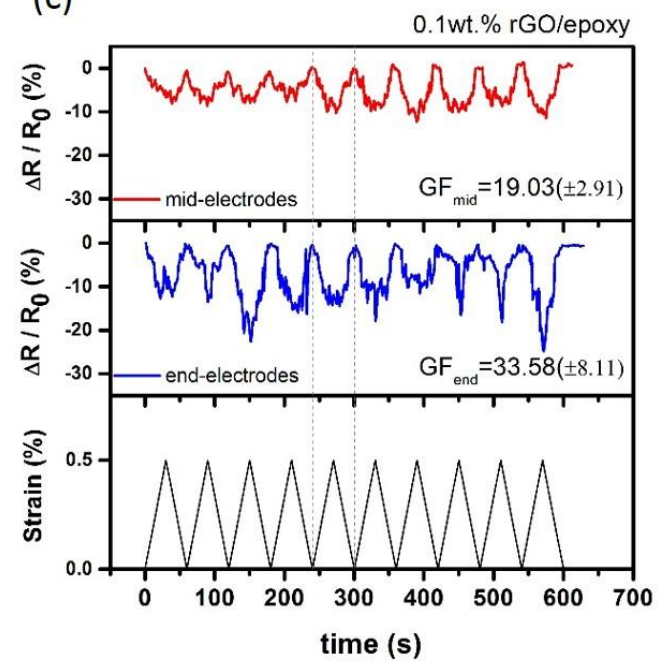

(b)

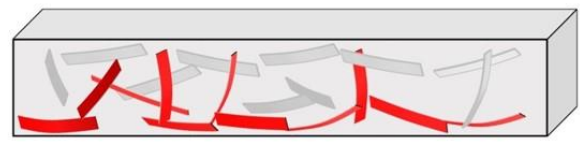

Three-dimensional rGO network under flexural loading

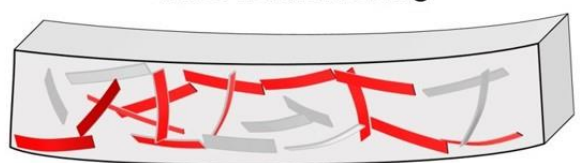

(d)

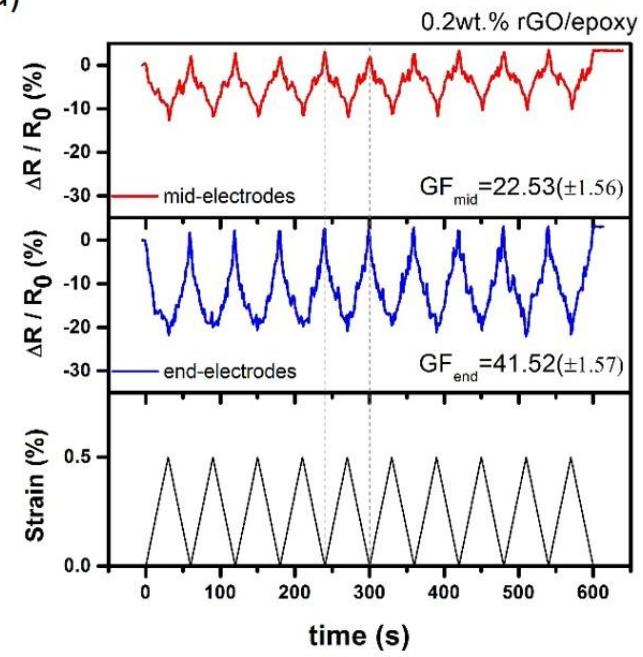

Fig. 6 Strain sensing results of rGO/epoxy nanocomposites under flexural loading: (a) the position of mid-(surface) and end electrodes; (b) illustration of the side-view of the internal 3D network elucidating the reduced resistance upon flexural loading due to reduced inter-particle distances between rGO in compression; (c) and (d) rGO/epoxy nanocomposites under cyclic loading with strains up to $0.5 \%$, showing high sensitivity (GF $=41.5)$ with a clear change in electrical resistance for each cycle.

\subsection{Joule heating based de-icing}

The de-icing capability of the fabricated $\mathrm{rGO} /$ epoxy nanocomposites was examined using the Ohmic or Joule heating effect for both a freezing environment and room temperature (RT) 
conditions (Fig. 7a). Good Joule heating capabilities were obtained for all 0.5 wt.\% samples, although a difference in clear heating efficiency was observed between high shear and medium shear TRM processed specimens as a result of differences in their internal nanofiller network structure. Very efficient heating was obtained for high shear mixed composites, requiring only $120 \mathrm{~s}$ to reach $20^{\circ} \mathrm{C}$ from freezing $\left(-20^{\circ} \mathrm{C}\right)$. Fig. $7 \mathrm{~b}$ shows the thermal image of a high shear mixed rGO/epoxy sample after 120 s heating from RT, confirming a homogeneous heat distribution within the panel.
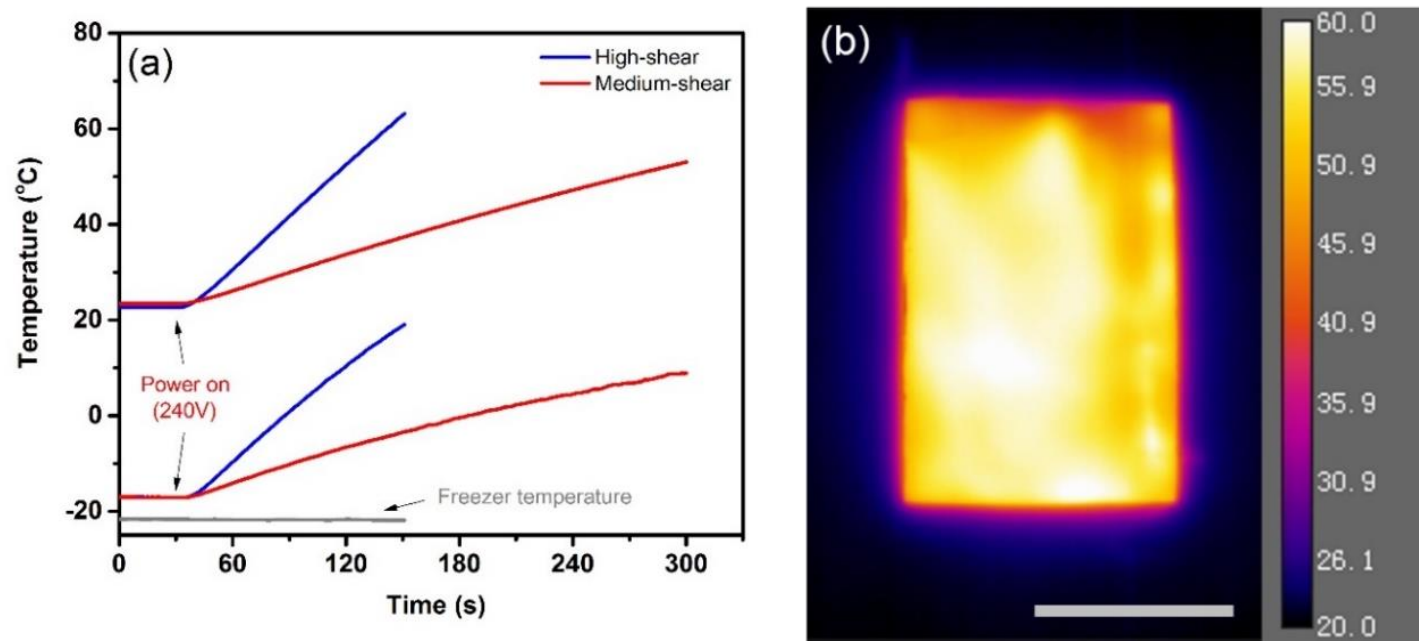

Fig. 7 Joule heating results of 0.5 wt.\% rGO/epoxy nanocomposites by both medium-and high shear TRM processing: (a) heating capability from freezing $\left(-20^{\circ} \mathrm{C}\right)$ and $R T$ environments; (b) thermal image of a high shear mixed nanocomposite sample after $120 \mathrm{~s}$ heating from RT, showing homogeneous heating of the sample (scale bar $=50 \mathrm{~mm}$ ).

\section{Conclusions}

The graphene network formation process in epoxy resin has been in-situ visualised and monitored under optical microscopy with different initial levels of nanofiller dispersion. The effect of network formation in epoxy based nanocomposites on electrical, mechanical, and other multifunctional properties such as strain sensing and Joule heating have been explored. Fracture surfaces have been examined to reveal the morphology of the final nanofiller network after curing. Nanofiller network formation time has been recorded and compared with previous literature for thermoplastic systems based on a simple viscosity correlation. 
Ultra-low electrical percolation thresholds have been obtained for rGO/epoxy nanocomposites with good levels of electrical conductivity $\left(10^{-1} \mathrm{~S} / \mathrm{m}\right)$ at low $(0.5$ wt.\%) filler loadings, while flexural modulus was increased by $12 \%$ with the addition of only $0.2 \mathrm{wt} . \%$ rGO. This efficient mechanical reinforcement is attributed to a homogeneous initial state of dispersion and a re-established network of $\mathrm{rGO}$ within epoxy resins after high shear three roll milling.

The strain sensing capability of the composites has been examined under flexural loadings using electrical methods, with high sensitivity (gauge factor 41 at $0.2 \mathrm{wt} . \% \mathrm{rGO}$ ) and good repeatability. Very efficient Joule heating with a potential application to de-icing was also successfully integrated in the nanocomposites through the percolated rGO network, with a heating capability from $-20^{\circ} \mathrm{C}$ to $20^{\circ} \mathrm{C}$ within $120 \mathrm{~s}$ under $240 \mathrm{~V}$.

This research not only presented direct evidence of the dynamic time-dependent reagglomeration process of graphene nanofillers within a liquid epoxy resin, but also provided a direct correlation with nanocomposite performances, providing important guidelines for nano-engineered epoxy based composites in order to tune their internal microstructures to achieve desired properties.

\section{Acknowledgement}

This work was carried out under the framework of the European Research Programme "PolyGraph" (grant agreement number 604143) in the Seventh Framework Programme.

\section{References:}

[1] Zha JW, Zhang B, Li RKY, Dang ZM. High-performance strain sensors based on functionalized graphene nanoplates for damage monitoring. Compos Sci Technol. 2016;123:32-8.

[2] Garlof S, Fukuda T, Mecklenburg M, Smazna D, Mishra YK, Adelung R, et al. Electro-mechanical piezoresistive properties of three dimensionally interconnected carbon aerogel (Aerographite)-epoxy composites. Compos Sci Technol. 2016;134:226-33.

[3] Jia J, Sun X, Lin X, Shen X, Mai Y-W, Kim J-K. Exceptional Electrical Conductivity and Fracture Resistance of 3D Interconnected Graphene Foam/Epoxy Composites. ACS Nano. 2014;8(6):5774-83.

[4] Chou T-W, Gao L, Thostenson ET, Zhang Z, Byun J-H. An assessment of the science and technology of carbon nanotube-based fibers and composites. Compos Sci Technol. 2010;70(1):1-19.

[5] Yousefi N, Sun X, Lin X, Shen X, Jia J, Zhang B, et al. Highly Aligned Graphene/Polymer Nanocomposites with Excellent Dielectric Properties for High-Performance Electromagnetic Interference Shielding. Adv Mater. 2014;26(31):5480-7. 
[6] Lu S, Tian C, Wang X, Zhang L, Du K, Ma K, et al. Strain sensing behaviors of GnPs/epoxy sensor and health monitoring for composite materials under monotonic tensile and cyclic deformation. Compos Sci Technol. 2018;158:94-100.

[7] Wei J, Vo T, Inam F. Epoxy/graphene nanocomposites - processing and properties: a review. RSC Advances. 2015;5(90):73510-24.

[8] Li Y, Zhang H, Crespo M, Porwal H, Picot O, Santagiuliana G, et al. In Situ Exfoliation of Graphene in Epoxy Resins: A Facile Strategy to Efficient and Large Scale Graphene Nanocomposites. ACS Appl Mater Interfaces. 2016;8(36):24112-22.

[9] Li W, Dichiara A, Bai J. Carbon nanotube-graphene nanoplatelet hybrids as high-performance multifunctional reinforcements in epoxy composites. Compos Sci Technol. 2013;74:221-7.

[10] Rafiee MA, Rafiee J, Wang Z, Song H, Yu Z-Z, Koratkar N. Enhanced Mechanical Properties of Nanocomposites at Low Graphene Content. ACS Nano. 2009;3(12):3884-90.

[11] Li Y, Zhang H, Porwal H, Huang Z, Bilotti E, Peijs T. Mechanical, electrical and thermal properties of in-situ exfoliated graphene/epoxy nanocomposites. Composites Part A: Applied Science and Manufacturing. 2017;95:229-36.

[12] Monti M, Rallini M, Puglia D, Peponi L, Torre L, Kenny JM. Morphology and electrical properties of graphene-epoxy nanocomposites obtained by different solvent assisted processing methods. Composites Part A: Applied Science and Manufacturing. 2013;46:166-72.

[13] Wu SY, Ladani RB, Zhang J, Bafekrpour E, Ghorbani K, Mouritz AP, et al. Aligning multilayer graphene flakes with an external electric field to improve multifunctional properties of epoxy nanocomposites. Carbon. 2015;94:607-18.

[14] Peijs T, Inam F, Wong DWY, Kuwata M. Multiscale hybrid micro-nanocomposites based on carbon nanotubes and carbon fibers. J Nanomater. 2010;2010(Article ID 453420):12.

[15] Zhang H, Bilotti E, Peijs T. The use of carbon nanotubes for damage sensing and structural health monitoring in laminated composites: a review. Nanocomposites. 2015;1(4):167-84.

[16] Zhang H, Liu Y, Kuwata M, Bilotti E, Peijs T. Improved fracture toughness and integrated damage sensing capability by spray coated CNTs on carbon fibre prepreg. Compos Pt A-Appl Sci Manuf. 2015;70:102-10.

[17] Li Y, Zhang H, Liu Y, Wang H, Huang Z, Peijs T, et al. Synergistic effects of spray-coated hybrid carbon nanoparticles for enhanced electrical and thermal surface conductivity of CFRP laminates. Compos Pt A-Appl Sci Manuf. 2018;105:9-18.

[18] Tang L-C, Wan Y-J, Yan D, Pei Y-B, Zhao L, Li Y-B, et al. The effect of graphene dispersion on the mechanical properties of graphene/epoxy composites. Carbon. 2013;60:16-27.

[19] Wan Y-J, Tang L-C, Yan D, Zhao L, Li Y-B, Wu L-B, et al. Improved dispersion and interface in the graphene/epoxy composites via a facile surfactant-assisted process. Compos Sci Technol.

2013;82:60-8.

[20] Inam F, Peijs T. Transmission light microscopy of carbon nanotubes-epoxy nanocomposites involving different dispersion methods. Adv Compos Lett. 2006;15(1):7-13.

[21] Inam FP, Ton. Re-agglomeration of carbon nanotubes in two-component epoxy system. Journal of Nanostructured Polymers and Nanocomposites. 2006;2(3):9.

[22] Zhang R, Dowden A, Deng H, Baxendale M, Peijs T. Conductive network formation in the melt of carbon nanotube/thermoplastic polyurethane composite. Compos Sci Technol. 2009;69(10):1499504.

[23] Zhang H, Bilotti E, Tu W, Lew CY, Peijs T. Static and dynamic percolation of phenoxy/carbon nanotube nanocomposites. Eur Polym J. 2015;68(0):128-38.

[24] Bilotti E, Zhang R, Deng H, Baxendale M, Peijs T. Fabrication and property prediction of conductive and strain sensing TPU/CNT nanocomposite fibres. Journal of Materials Chemistry. 2010;20(42):9449-55.

[25] Battisti A, Skordos AA, Partridge IK. Monitoring dispersion of carbon nanotubes in a thermosetting polyester resin. Compos Sci Technol. 2009;69(10):1516-20.

[26] Prolongo SG, Jimenez-Suarez A, Moriche R, Ureña A. In situ processing of epoxy composites reinforced with graphene nanoplatelets. Compos Sci Technol. 2013;86:185-91. 
[27] Chandrasekaran S, Seidel C, Schulte K. Preparation and characterization of graphite nanoplatelet (GNP)/epoxy nano-composite: Mechanical, electrical and thermal properties. Eur Polym J. 2013;49(12):3878-88.

[28] Bilotti E, Zhang H, Deng H, Zhang R, Fu Q, Peijs T. Controlling the dynamic percolation of carbon nanotube based conductive polymer composites by addition of secondary nanofillers: The effect on electrical conductivity and tuneable sensing behaviour. Compos Sci Technol. 2013;74:85-90.

[29] Zhang H, Liu Y, Huo S, Briscoe J, Tu W, Picot OT, et al. Filtration effects of graphene nanoplatelets in resin infusion processes: Problems and possible solutions. Compos Sci Technol. 2017;139:138-45. [30] Inam F, Reece MJ, Peijs T. Shortened carbon nanotubes and their influence on the electrical properties of polymer nanocomposites. J Compos Mater. 2011;46(11):1313-22.

[31] Martin-Gallego M, Bernal MM, Hernandez M, Verdejo R, Lopez-Manchado MA. Comparison of filler percolation and mechanical properties in graphene and carbon nanotubes filled epoxy nanocomposites. Eur Polym J. 2013;49(6):1347-53.

[32] Stankovich S, Dikin DA, Dommett GHB, Kohlhaas KM, Zimney EJ, Stach EA, et al. Graphene-based composite materials. Nature. 2006;442(7100):282-6.

[33] S. WA, Tanvir AHS, Sriya D, Fahmida I, F. JA, J. GM. High - Performance Pristine Graphene/Epoxy Composites With Enhanced Mechanical and Electrical Properties. Macromol Mater Eng.

2013;298(3):339-47.

[34] Sellam C, Zhai Z, Zahabi H, Picot OT, Deng H, Fu Q, et al. High mechanical reinforcing efficiency of layered poly(vinyl alcohol) - graphene oxide nanocomposites. Nanocomposites. 2015;1(2):7.

[35] Suk JW, Piner RD, An J, Ruoff RS. Mechanical Properties of Monolayer Graphene Oxide. ACS Nano. 2010;4(11):6557-64.

[36] Lee C, Wei X, Kysar JW, Hone J. Measurement of the Elastic Properties and Intrinsic Strength of Monolayer Graphene. Science. 2008;321(5887):385-8.

[37] Chatterjee S, Nafezarefi F, Tai NH, Schlagenhauf L, Nüesch FA, Chu BTT. Size and synergy effects of nanofiller hybrids including graphene nanoplatelets and carbon nanotubes in mechanical properties of epoxy composites. Carbon. 2012;50(15):5380-6.

[38] Shen X-J, Liu Y, Xiao H-M, Feng Q-P, Yu Z-Z, Fu S-Y. The reinforcing effect of graphene nanosheets on the cryogenic mechanical properties of epoxy resins. Compos Sci Technol. 2012;72(13):1581-7.

[39] Ghaleb ZA, Mariatti M, Ariff ZM. Properties of graphene nanopowder and multi-walled carbon nanotube-filled epoxy thin-film nanocomposites for electronic applications: The effect of sonication time and filler loading. Composites Part A: Applied Science and Manufacturing. 2014;58:77-83. [40] Pathak AK, Borah M, Gupta A, Yokozeki T, Dhakate SR. Improved mechanical properties of carbon fiber/graphene oxide-epoxy hybrid composites. Compos Sci Technol. 2016;135:28-38.

[41] Zhang H, Liu Y, Bilotti E, Peijs T. In-situ monitoring of interlaminar shear damage in carbon fibre composites. Adv Compos Lett. 2015;24(4):92-7.

[42] Moriche R, Sánchez M, Jiménez-Suárez A, Prolongo SG, Ureña A. Strain monitoring mechanisms of sensors based on the addition of graphene nanoplatelets into an epoxy matrix. Compos Sci Technol. 2016;123:65-70. 